\title{
Relation Between Plasma Glucose Levels of Mother and Fetus During Maternal Hyperglycemia, Hypoglycemia, and Fasting in the Rat
}

\author{
Charles J. Goodner ${ }^{[17]}$, Martin J. Conway, and Jon H.Werrbach \\ The Robert H.Williams Laboratory for Clinical Investigation, the Department of Medicine, \\ King County Harborview Hospital, and the University of Washington School of Medicine, \\ Seattle, Washington, USA
}

Extract

The relation between the concentration of glucose in maternal and fetal plasma was examined in pregnant rats during the last three days of gestation. Rats were studied after being fasted for 16 hours following two hours of infusion of physiologic saline or insulin $(1,000 \mu \mathrm{U} /$ minute $)$ in either $30 \%$ glucose or saline. After a constant concentration of glucose in maternal plasma had been achieved, the rats were sacrificed rapidly and fetal blood was obtained. In fasting animals, before day 20, the concentration of glucose in fetal plasma was low, and a gradient of 2.0 or greater prevailed between maternal and fetal plasma glucose. This gradient fell rapidly during days 20 and 21 to average 1.0 for the last 24-48 hours before term. In the fetus, the rise in the level of glucose paralleled that of hepatic glycogen. When maternal hyperglycemia was induced by infusion of glucose, the gradient in the younger fetuses was poorly maintained and fetal hyperglycemia occurred at all ages. When maternal hypoglycemia was induced by infusion of insulin, the younger fetuses displayed proportional hypoglycemia; however, after day 20, the level of plasma glucose in the fetus exceeded the maternal level, and the placental gradient for glucose was reversed.

It is concluded that during maternal hypoglycemia, the near-term fetus is capable of independently regulating the level of glucose in blood for at least two hours. During maternal hyperglycemia, the normal placental gradient for glucose characteristic of the younger fetus fails to prevent completely fetal hyperglycemia, and near-term, maternal hyperglycemia is transmitted to the fetus without evident fetal counter regulation.

\section{Speculation}

This study provides additional evidence for the thesis that the near-term fetus develops independent mechanisms for production and regulation of glucose during periods of glucose deprivation. The development and use of such mechanisms in utero during maternal fasting may represent an important preparation for extrauterine existence. It remains to be determined whether the prevailing hyperglycemia of diabetes during gestation might delay development of the pathways involved. 


\section{Introduction}

Regulation of glucose levels in blood is a complex process involving the endocrine system, hepatic metabolism, and the gastrointestinal tract. In utero, the fetus receives glucose from maternal plasma, but immediately after birth must institute its own production and regulation of glucose in order to survive. Previous studies from this laboratory [8] have suggested that during maternal fasting, glucose in fetal plasma derives in part from fetal hepatic gluconeogenesis. These findings, based upon kinetic studies using $\mathrm{C}^{14}$ glucose, led to the postulation that the fetus participates in regulation of plasma glucose in utero in preparation for extrauterine life. The present studies demonstrate that during maternal hypoglycemia, the level of glucose in fetal blood is higher than that in maternal blood. This finding provides independent evidence for the hypothesis that fetal mechanisms participate in the regulation of the levels of glucose in fetal blood in utero during periods of relative glucose deprivation. Examination of the relation between the levels of maternal and fetal plasma glucose during maternal hyperglycemia, however, indicates that the ability of the fetus to counteract an acute excessive delivery of maternal glucose is limited.

\section{Methods}

Rats of the Sprague-Dawley strain were mated during a 12-hour period and studied from the 18th to 22nd day of gestation by techniques previously described [8]. All rats were fasted 16 hours before study, anesthetized lightly with pentobarbital ( $3 \mathrm{mg} / 100 \mathrm{~g}$ body weight injected intraperitoneally), and infused intravenously with saline containing $\mathrm{C}^{14}$ labeled glucose or amino acid in tracer quantities for kinetic studies [7]: Glucose-loaded rats were given infusion and loading solutions containing $30 \%$ glucose. Insulin-treated rats received infusion solutions containing $50 \mathrm{mU} / \mathrm{ml}$ crystalline bovine insulin. Infusions by syringe pump were at the rate of $1.2 \mathrm{ml}$ per hour. A loading dose of $1.0 \mathrm{ml}$ infusion solution was administered first.

During the course of infusion studies, five fasting control rats spontaneously developed apnea and cyanosis. These animals were immediately sacrificed and studied as examples of the effect of anoxia upon maternal:fetal glucose relations.

The concentration of glucose in maternal plasma (tail blood) was measured at 30-minute intervals during the infusion to document the stability of the experimental conditions (see fig. 1). After two hours of infusion, the rats were rapidly sacrificed by two operators who collected maternal blood and liver and duplicate pools of fetal blood, fetal liver, and placenta. The pool

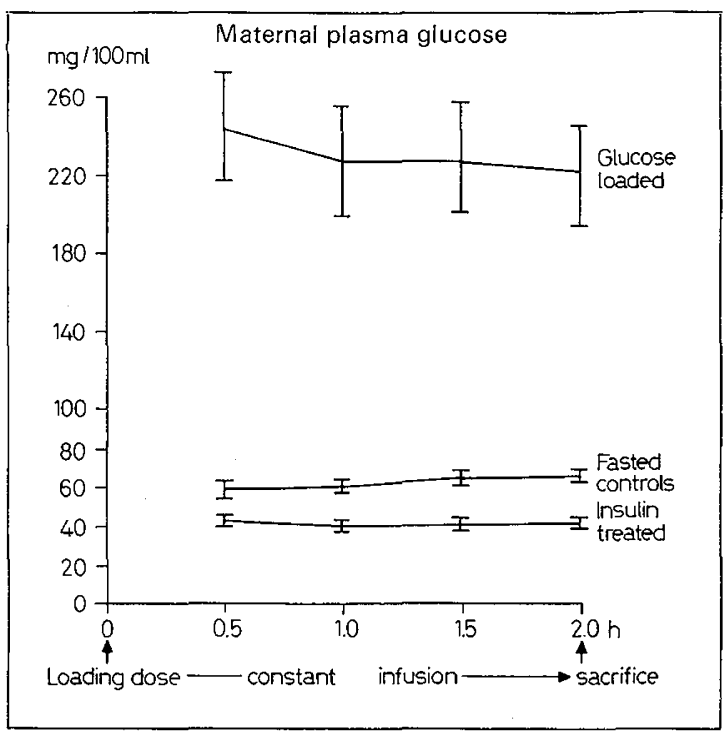

Fig. 1. Pregnant rats were fasted 16 hours, anesthetized, and infused with saline (control fasted rats), $30 \%$ glucose (glucose-loaded rats), or insulin $1000 \mu \mathrm{U} / \mathrm{min}$ (insulin-treated rats). Tail blood was collected and analyzed for glucose at 30-minute intervals. The brackets include two standard errors of the mean. Fasted group, 22 rats; glucose-loaded, 10 rats; insulin-treated, 12 rats.

of fetal material from each operator represented accumulations from three fetuses. Concentrations of glucose in maternal and fetal plasma were determined with glucose oxidase using deproteinized filtrates. The glucose determinations on duplicate pools of fetal plasma agreed closely in most instances. The coefficient of variation for the glucose method on replicate samples of plasma was $4 \%$ at $56 \mathrm{mg} / 100 \mathrm{ml}(\mathrm{n}=9)$. Liver and placental glycogen was determined by the method of GOOD [5].

\section{Results}

\section{Glycogen Content in Relation to Growth of Placenta and Fetal Liver}

Figure 2A illustrates the relative size of the fetal liver and placenta during the last 4 days of gestation. The placenta grows little during this phase of gestation, while liver increases rapidly in weight until term. The glycogen content of these two organs is illustrated in figure $2 \mathrm{~B}$. Placental glycogen is low throughout this period in spite of the larger mass of this organ. The glycogen content in liver begins to increase abruptly at fetal weight above 3 grams. The period of maximum change in hepatic glycogen is during the 20th day of gestation when fetal weight is increasing from 3 to 4 grams. 


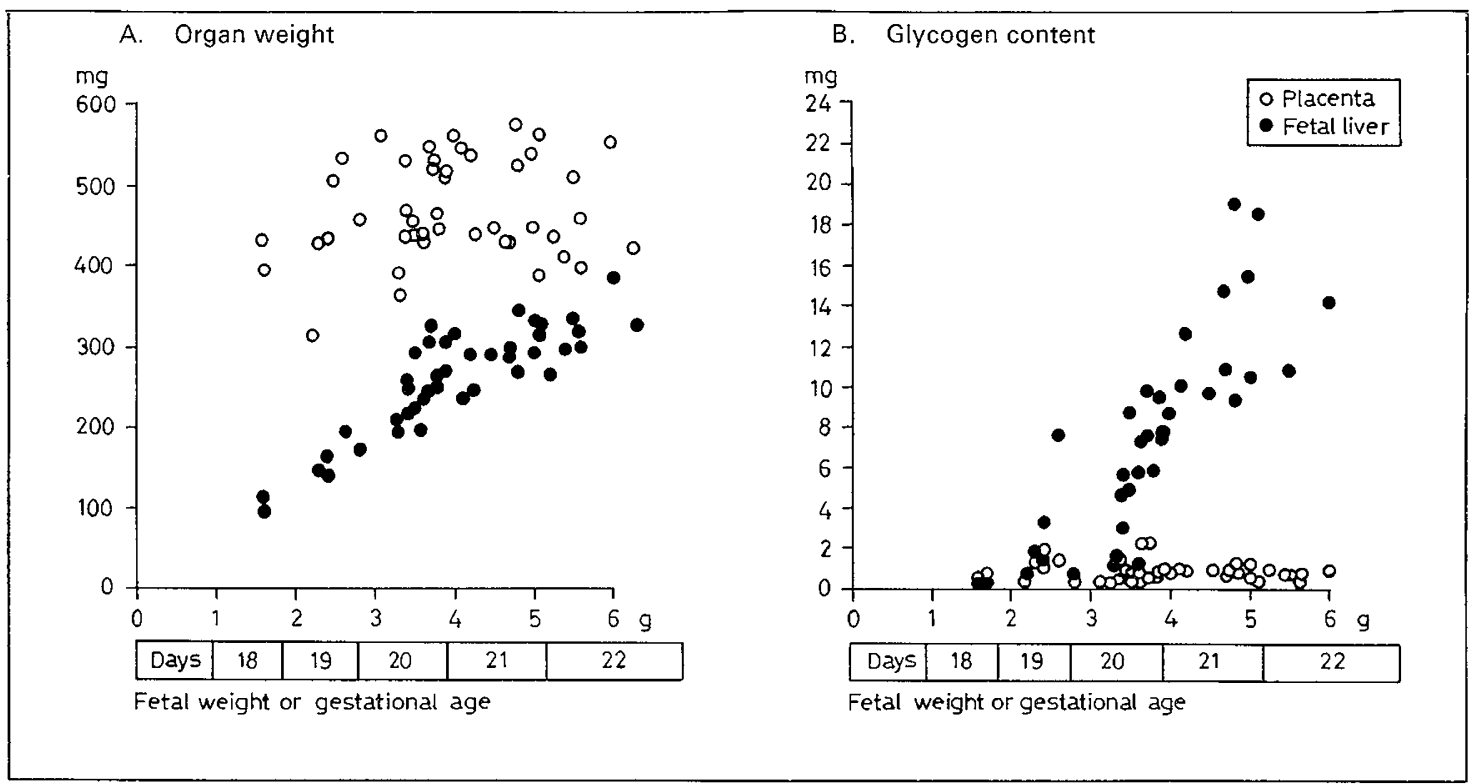

Fig. 2. A. Relation between the gestational age or fetal weight and the weight of placenta and fetal liver in fasted control rats. $B$. Relation between the gestational age or fetal weight and the glycogen content of the placenta and fetal liver in fasted control rats.

Fig. 3. A. Relation between the gestational age or fetal weight and the concentration of glucose in pooled fetal plasma at sacrifice in fasted control rats. $B$. Relation between maternal and pooled fetal plasma glucose in fasted control rats as a function of gestational age or fetal weight.
Relation of Glucose in Fetal Plasma to that of Maternal Plasma Figure $3 \mathrm{~A}$ presents the concentration of fetal plasma in glucose in fetuses whose mothers were fasted for 16 hours during the last 4 days of gestation. Like the glycogen content in liver, the level of glucose rises abruptly as the fetus grows from 3 to 4 grams. In fetuses weighing less than 3 grams, the level is below $30 \mathrm{mg} / 100 \mathrm{ml}$. Figure $3 \mathrm{~B}$ illustrates the changing relation between levels of glucose in maternal and fetal plasma during the same period. Levels in maternal plasma did not vary significantly with time during this pericd, averag-

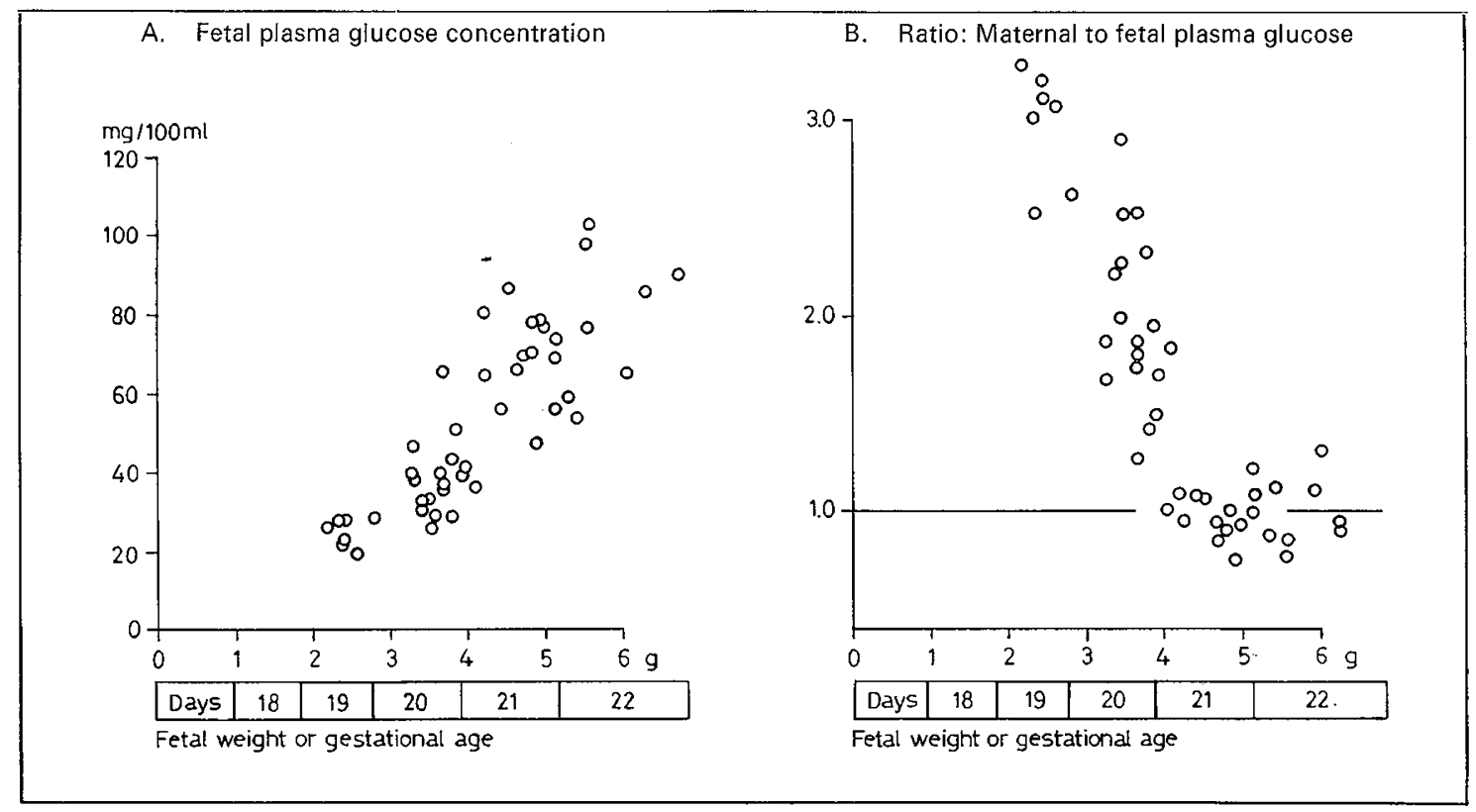


ing $66 \pm 1.6(\mathrm{SE}) \mathrm{mg} / 100 \mathrm{ml}$. The ratio of maternal to fetal glucose in plasma changed markedly. It was above 3.0 in fetuses weighing less than 3 grams, dropped rapidly toward 1.0 in fetuses weighing between 3 and 4 grams, and averaged 1.0 in fetuses weighing between 4 grams and term.

The Effect of Maternal Hyperglycemia upon Glucose in Fetal Plasma

When sustained hyperglycemia was induced in the mother by infusion of $30 \%$ glucose for two hours, the level of glucose in fetal plasma rose significantly regardless of gestational age. Results in 10 rats with fetal weights varying from 2.5 to 6.3 grams are listed in table I. The maternal to fetal ratio for plasma glucose tended to be higher in rats of earlier gestational age, but never exceeded 2.0. Thus, in the presence of maternal hyperglycemia, the high maternal-fetal gradient of the younger fetus was not completely maintained and fetal hyperglycemia was easily induced. The glycogen content of fetal liver was not increased by the two-hour infusion of glucose; however, application of isotopic techniques [8] indicated that glycogen synthesis was accelerated.

The Effect of Maternal Hypoglycemia upon Glucose in Fetal Plasma

When hypoglycemia was induced and maintained for two hours in the mother by infusion of $1000 \mu \mathrm{U}$ insulin/minute, the concentration of glucose in fetal plasma was significantly higher than that in the maternal plasma in 7 of 9 fetuses weighing more than $4.5 \mathrm{~g}$.

Table I. Glucose-loaded rats

\begin{tabular}{|c|c|c|c|c|c|}
\hline \multirow[b]{2}{*}{$\begin{array}{l}\text { s. } \\
\text { on }\end{array}$} & \multicolumn{2}{|c|}{$\begin{array}{c}\text { Glucose levels } \\
\text { in plasma }\end{array}$} & \multirow{2}{*}{ 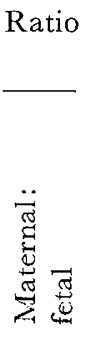 } & \multicolumn{2}{|c|}{$\begin{array}{l}\text { Fetal liver } \\
\text { glycogen }\end{array}$} \\
\hline & 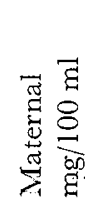 & 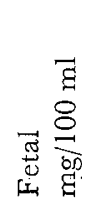 & & 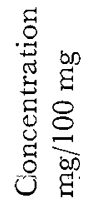 & 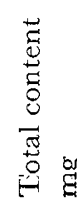 \\
\hline 2.5 & 150 & 125 & 1.2 & 2.0 & 3.7 \\
\hline 3.4 & 372 & 233 & 1.6 & (). 3 & 0.7 \\
\hline 3.5 & 1.58 & 95 & 1.7 & 3.0 & 15 \\
\hline 3.9 & 246 & 191 & 1.3 & (6.7 & 21 \\
\hline 4.1 & 232 & 134 & 1.7 & 4.3 & 12 \\
\hline 4.2 & 320 & 300 & 1.1 & 1.7 & 4.7 \\
\hline 4.9 & 1888 & 140 & 1.3 & 2.4 & 7.5 \\
\hline 5.2 & 184 & 163 & 1.1 & 1.5 & 5.6 \\
\hline 5.7 & 178 & 158 & 1.2 & 3.3 & 10 \\
\hline 6.3 & 122 & 94 & 1.3 & 6.6 & 24. \\
\hline
\end{tabular}

Table II. Insulin-treated rats

\begin{tabular}{|c|c|c|c|c|c|}
\hline \multirow[b]{2}{*}{ 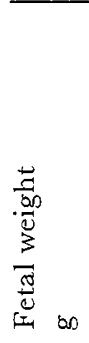 } & \multicolumn{2}{|c|}{$\begin{array}{c}\text { Glucose levels } \\
\text { in plasma }\end{array}$} & \multirow{2}{*}{ 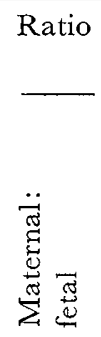 } & \multicolumn{2}{|c|}{$\begin{array}{l}\text { Fetal liver } \\
\text { glycogen }\end{array}$} \\
\hline & 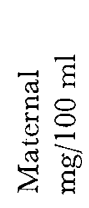 & 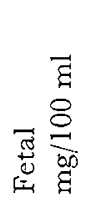 & & 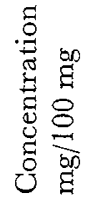 & 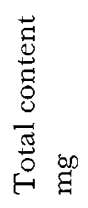 \\
\hline 2.5 & 44 & 13 & 3.4 & 0.70 & 1.6 \\
\hline 3.2 & 50 & 26 & 1.9 & 3.1 & 6.5 \\
\hline 4.3 & 40 & 20 & 2.0 & 4.0 & 11 \\
\hline 4.8 & 48 & 75 & 0.64 & 2.3 & 5.7 \\
\hline 4.9 & 39 & 46 & 0.85 & 5.4 & 16 \\
\hline 5.0 & 46 & 61 & 0.75 & 3.6 & 9.5 \\
\hline 5.0 & 40 & 39 & 1.03 & 5.3 & 13 \\
\hline 5.0 & 43 & 55 & 0.78 & 3.5 & 12 \\
\hline 5.4 & 39 & 70 & 0.56 & 2.8 & 8.5 \\
\hline 5.5 & 46 & 49 & 0.94 & 4.2 & 10 \\
\hline 5.9 & 33 & 48 & 0.69 & 4.1 & 10 \\
\hline 6.4 & 46 & 86 & 0.54 & 5.1 & 14 \\
\hline
\end{tabular}

Table III. Anoxic rats

\begin{tabular}{|c|c|c|c|c|c|}
\hline \multirow[b]{2}{*}{ 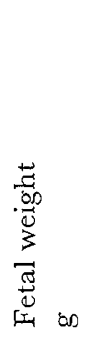 } & \multicolumn{2}{|c|}{$\begin{array}{l}\text { Glucose levels } \\
\text { in plasma }\end{array}$} & Ratio & \multicolumn{2}{|c|}{$\begin{array}{l}\text { Fetal liver } \\
\text { glycogen }\end{array}$} \\
\hline & 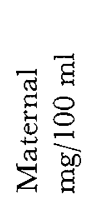 & 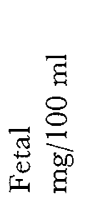 & 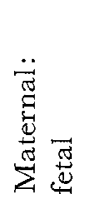 & 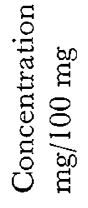 & 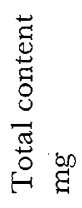 \\
\hline 4.0 & 118 & 119 & 1.0 & 2.5 & 6.9 \\
\hline 4.3 & 174 & 127 & 1.4 & 2.7 & 8.9 \\
\hline 4.4 & 63 & 112 & 0.56 & 3.0 & 7.6 \\
\hline 5.0 & 108 & 153 & 0.71 & 3.7 & 12 \\
\hline 5.3 & 126 & 146 & 0.86 & 2.6 & 6.5 \\
\hline
\end{tabular}

Table IV. Glycogen concentration in maternal liver and placenta in the three experimental groups ${ }^{1}$

\begin{tabular}{lccc}
\hline Group & $\begin{array}{c}\text { Maternal } \\
\text { liver }\end{array}$ & Placenta & $\mathrm{n}$ \\
\hline 16-hour fasted control & $0.55+0.12$ & $0.11+0.04$ & 22 \\
Glucose-loaded & $2.10+(0.70$ & $0.30+0.06$ & 10 \\
Insulin-treated & $0.28+0.28$ & $0.08+0.01$ & 12
\end{tabular}

1 Values given are mean and standard deviation of the mean. 
Table II lists the concentrations and the maternal-fetal ratio for glucose in 12 insulin-treated rats. In the three fetuses weighing less than 4.5 grams, the expected maternal-fetal gradient was maintained.

In the insulin-treated rats, the glycogen content of fetal liver was not greatly reduced from the expected values in untreated rats. The store of glycogen in fetal liver, however, was very large compared with the glucose pool; hence, glycogenolysis may have contributed to maintenance of glucose levels during the 2-hour period without being detected by chemical measurement of glycogen content in fetal liver.

\section{Maternal-Fetal Glucose Gradient during the Endogenous Hyperglycemia of Anoxia}

Five fasting control rats developed apnea and cyanosis during the last hour of infusion experiments and were sacrificed as quickly as possible. Table III lists the concentrations and ratio of the levels of glucose in maternal and fetal plasma. Anoxia resulted in hyperglycemia in the mother or the fetus or both. In three of five determinations, the level of glucose in fetal plasma significantly exceeded that in maternal plasma, in marked contrast to findings with exogenously induced maternal hyperglycemia (table I), in which the level of glucose in maternal plasma always exceeded that in fetal plasma.

The Effects of Glucose Loading and Insulin-Induced Hypoglycemia upon Stores of Glycogen in Maternal Liver and Placenta

Table IV presents the mean values for concentration of glycogen in maternal liver and placenta in the three experimental groups. Glucose loading for two hours significantly increased glycogen content in both organs (p 0.01). Insulin-induced hypoglycemia for the same length of time significantly reduced glycogen content in maternal liver ( $p 0.02)$, but that in the placenta was unchanged.

\section{Discussion}

There has been disagreement regarding the concentration of glucose in fetal plasma during gestation [13]. Species differences, artifacts of analysis, and differences in the degree of anoxia induced in collecting fetal blood have been cited to explain the discrepancies. The technique of sacrifice employed in the present study was developed to minimize the period of interrupted placental circulation but obviously was not as physiologic as methods of direct sampling in utero available in larger species [2]. Within the limitations of technique, however, the developmental pattern of regulation of glucose in fetal blood of the rat appears to be one of change from a high maternal-fetal gradient in younger fetuses to no gradient as term is reached on days 21 and 22. The rise in the level of glucose in the fetus to that in the adult coincides with the development of the large glycogen store in fetal liver characteristic of late gestation in mammalian species [12]. This rise also corresponds to the period of rapid maturation of hepatic enzymes for glycogen synthesis [1]. Some evidence suggests that during this same period (19-21 days), fetal tissues develop sensitivity to insulin [10], while the appearance of insulin in the pancreas of the rat fetus has been reported to occur on the 14th day of gestation [4]. The high maternal-fetal gradient for glucose in fetuses weighing less than 4.0 grams cannot be ascribed to limited placental mass, since the placenta is nearly maximal in size before this stage of gestation and the ratio of placental to fetal weight is actually greater than during later stages. Developmental changes in placental transport systems for glucose could produce the changing gradient.

It is possible that the rising level of glucose in fetal blood in later gestation reflects the development of mechanisms for independent regulation of glucose concentration by the fetus. Our previous studies, suggesting active gluconeogenesis within the conceptus in utero, add support to this possibility [8]. The behavior of glucose in fetal blood during maternal hyperglycemia, however, does not suggest independent regulation of glucose in fetal blood but, rather, direct transmission of the maternal glucose to the fetus through a placental transport system. The parallel behavior of glucose in fetal and maternal plasma during glucose loading has led to the general conclusion that glucose in fetal plasma is passively determined by the maternal concentration [14].

In the present study, the behavior of glucose in fetal plasma during maternal hypoglycemia is not compatible with this hypothesis. The concentration of glucose in maternal plasma was documented to be stable at the hypoglycemic level for at least 90 minutes before sacrifice (fig. 1). Thus a new gradient would have had ample time to be established. The higher concentration in the fetus at sacrifice therefore represents a qualitative reversal of the usual relation and is not a transient artifact of disequilibrium. The fetal-maternal gradient at sacrifice implies a net flow of glucose from the fetus to the mother during maternal hypoglycemia.

These data indicate that the fetus is capable of regulating glucose levels in blood independently of the maternal circulation, at least during short-term observations. The source of glucose in the fetus during maternal hypoglycemia could be either glycogenolysis or gluconeogenesis in fetal liver or both. A contribution of placental glycogen to fetal plasma glucose might have occurred, but would not have been quantitatively im- 
portant, since the net change in placental glycogen was small compared with estimated fetal utilization. Either mechanism mentioned above would of necessity be effective for only a finite period, since both require consumption of limited fetal substrates. During gluconeogenesis, if amino acids were to constitute the major glucose precursor, the induction of maternal hypoglycemia by insulin would heighten the competition for amino acids among maternal tissues, fetal liver, and growing fetal tissues.

Glycogenolysis by fetal liver in response to hypoglycemia in the mother would presumably involve activation of phosphorylase. Activity of this enzyme was not demonstrated in these experiments and no conclusion regarding this enzyme appears justified.

During acute severe anoxia following maternal apnea, it would appear that both maternal and fetal glycogenolytic systems were activated, since levels of glucose in fetal and maternal blood did not follow the gradient pattern expected from the primary elevation in the level of glucose in maternal blood. During less severe anoxia, lasting 30-50 minutes, Thalme [11] found maternal hyperglycemia without obvious fetal hyperglycemia. The level of fetal lactate, however, rose markedly. DAwEs [3] previously demonstrated that anoxia is a potent stimulus to glycogenolysis in fetal lamb liver and cardiac muscle.

The age of the fetus influenced the effects of altered concentration of glucose in maternal blood upon that in the fetus. During maternal hyperglycemia, the younger fetuses were partially protected by virtue of the higher placental gradient before day 21 ; however, the high gradient present during fasting was not fully maintained and the level of glucose was higher even in the youngest fetuses.

During maternal hypoglycemia, the higher placental gradient of the younger fetuses was maintained and the level of glucose was correspondingly low, whereas in fetuses weighing more than 4.5 grams (days 21 and 22) the level in fetal blood became higher than that in maternal blood.

Previous studies, in which labeled insulin was used, demonstrated negligible transplacental passage of insulin from mother to fetus [6]. The fetal responses to insulin-induced maternal hypoglycemia reported in this study are compatible with those of the earlier studies since hypoglycemia did not occur within the fetus. A possible effect of maternal insulin upon the glucose transport system in the placenta cannot be excluded, but results of concurrent studies of glucose kinetics do not suggest such an effect [7].

The ability of the older fetus to regulate the level of glucose in blood when maternal hypoglycemia is present may explain the clinical observation that hypoglycemic reactions in pregnant diabetic patients are rarely associated with fetal loss [9]. Prolonged hypoglycemia, however, might be expected to lead to exhaustion of glycogen stores and gluconeogenetic precursors and a decrease of anabolic processes. At earlier stages of gestation, when glycogen stores are limited, such effects would occur more quickly. Thus, in spite of the placental barrier precluding direct effects of maternal insulin within the fetus, repeated or prolonged maternal hypoglycemia might induce developmental defects through these indirect mechanisms.

\section{References and Notes}

1. Ballard, F.J. and Olrver, I.T. : Glycogen metabolism in embryonic chick and neonatal rat liver. Biochim. biophys. Acta 71: 578 (1963).

2. Battaglia, F. C.; Hellegers, A. E.; Heller, C.J. and BEHRMAN, R. : Glucose concentration gradients across the maternal surface, the placenta and the amnion of the rhesus monkey (Macaca mulatta). Amer. J. Obstet. Gynec. 88: 32 (1964).

3. Dawes, G.S.; Mott, J.C. and Shelley, H.J.: The importance of cardiac glycogen for maintenance of life in foetal lambs and new-born animals during anoxia. J. Physiol. 146: 516 (1959).

4. Dixit, P.K.; Lowe, I.P.; Heggestad, C. B. and LAzArow, A.: Insulin content of microdissected fetal islets obtained from diabetic and normal rats. Diabetes 13: 71-77 (1964).

5. Good, C. A.; Kramer, H. and Somogyi, M.: The determination of glycogen. J. biol. Chem. 100: 485 (1933).

6. Goodner, C.J. and Freinkel, J.: Carbohydrate metabolism in pregnancy. IV. Studies on the permeability of the rat placenta to $\mathrm{I}^{131}$ insulin. Diabetes 10: 383 (1961).

7. Goodner, C.J.; Conway, M.J. and Werrbach, J.H. : Glucose metabolism in the fetus in utero: The effect of maternal hypoglycemia during insulin infusion in the rat. Unpublished data.

8. Goodner, C.J. and Thompson, D.J.: Glucose metabolism in the fetus in utero: The effect of maternal fasting and glucose loading in the rat. Pediat. Res. 1: 443-451 (1967).

9. Hagbard, L.: Pregnancy and diabetes mellitus; in: American lecture series No.449 (ed. Hamblen, E. C.), chapt. 5 (Thomas, Springfield, Ill. 1961).

10. Prcon, L.: Effect of insulin on growth and biochemical composition of the rat fetus. Endocrinology 81: 1419-14 (1967).

11. Thalme, B.: Electrolyte and acid-base balance in fetal and maternal blood. Acta obstet.gynec. scand. V.45, suppl. 8 (1966).

12. Shellex, H. J.: Glycogen reserves and their 
mother and fetus during maternal hyperglycemia, hypoglycemia, and fasting in the rat

changes at birth and in anoxia. Brit. med. Bull. 17 : 137 (1961).

13. Shelley, H.J. and Neligan, G.A.: Neonatal hypoglycemia. Brit. med. Bull. 22: 34 (1966).

14. WIDDAs, W.F.: Transport mechanisms in the foetus. Brit. med. Bull. 17: 107 (1961).

15. The authors are indebted to Miss Mary Ann Berry and Mr. Edward Miller for their excellent technical assistance with these investigations.

16. This investigation was supported in part by Re- search Grant HD 00405, AM 10866, and Training Grant AM 5331 from the United States Public Health Service. The work was performed under the tenure of a USPHS Research Fellowship Grant AM 33-182, Dr. Conway, and a Lederle Medical Faculty Award, Dr. Goodner.

17. Requests for reprints should be addressed to: Charles J. Goodner, M.D., King County Harbroview Hospital, 325 Ninth Avenue, Seattle, Wash. 98104 (USA). 